Pacific Journal of Mathematic 


\title{
THE UNIQUENESS OF ELONGATIONS OF ABELIAN GROUPS
}

\author{
R. B. WARFIELD, JR.
}

Given an Abelian group $G$ and a functorial characteristic subgroup $h G$, we study the extent to which $G$ is determined up to isomorphism by $h G$ and $G / h G$. If $G$ is a $p$-group or a mixed module over a discrete valuation ring, we study the structure of $G$ in terms of that of $p^{\lambda} G$ and $G / p^{\lambda} G$, where $\lambda$ is a limit ordinal. We also study a corresponding family of subgroups of Abelian groups in general. For a countably generated reduced module $M$ of finite torsion-free rank over the ring $Z_{p}$ of integers localized at $p$, we obtain necessary and sufficient conditions for $M$ to be determined up to isomorphism by $p^{\lambda} M$ and $M / p^{2} M$.

1. Introduction. It is a famous result of Leo Zippin's [18], that if $G$ and $H$ are countable $p$-groups, $\lambda$ is a limit ordinal, $\phi: p^{\lambda} G \rightarrow$ $p^{\lambda} H$ is an isomorphism, and $G / p^{\lambda} G \cong H / p^{\lambda} H$, then $\phi$ extends to an isomorphism of $G$ onto $H$. Crawley [1] and Hill and Megibben [7] improved this result by omitting the countability condition on $G$ and $H$ but requiring that $\lambda$ be a countable limit ordinal and that $G / p^{\lambda} G$ be a direct sum of countable groups. (The countability condition on $\lambda$ can now be omitted by requiring that $G / p^{2} G$ be totally projective.) In this paper we prove several extensions and converses for these results, for uncountable $p$-groups and for countable mixed groups of finite torsion-free rank.

In $\S 3$, it is shown that if $A$ is a $p$-group with $p^{\omega} A=0$, and $A$ is not a direct sum of cyclic groups, then there are nonisomorphic p-groups $G$ and $H$ (which can be chosen of length $\omega+1$ ) such that $p^{\omega} G \cong p^{\omega} H$, and $G / p^{\omega} G \cong H / p^{\omega} H \cong A$. This strengthens a recent result of Nunke's [10, Theorem 3.4]. We call a pair $(A, B)$ of $p$ groups a uniquely $\lambda$-elongating pair if (i) there exists a reduced $p$ group $G$ such that $p^{\lambda} G \cong B$ and $G / p^{\lambda} G \cong A$, (ii) $B \neq 0$, and (iii) any two such groups are isomorphic. We follow Pierce [11] in calling a group $G$ of this type a $\lambda$-elongation of $A$ by $B$. A consequence of the results of Crawley and Hill and Megibben mentioned earlier is that if $A$ is a direct sum of cyclic $p$-groups, then $(A, B)$ is a uniquely $\omega$-elongating pair for any group $B$ for which $\omega$-elongations of $A$ by $B$ exist. It is conjectured that a strong converse holds, which would say that if $(A, B)$ is a uniquely $\omega$-elongating pair of $p$-groups, then $A$ is a direct sum of cyclic groups. In Theorem 3.2, we prove this under the additional assumption that $A / p A$ is countable, and assuming the continuum hypothesis. 
The rest of the paper is concerned with countable Abelian groups of finite torsion-free rank. We work primarily with modules over the ring $Z_{p}$ of integers localized at $p$. (This is the ring of those rational numbers which can be written as fractions with denominator prime to $p$. We recall that the category of $Z_{p}$-modules can be identified with the category of all Abelian groups $G$, such that for all primes $q$ different from $p$, multiplication by $q$ is an automorphism of $G$.)

If $\lambda$ is a limit ordinal, we say a module $A$ has the $\lambda$-Zippin property if (i) $\lambda$ is the length of the torsion subgroup $t(A)$, (ii) $p^{2} A=$ 0 , and (iii) given two modules $G$ and $H$ such that $G / p^{2} G \cong H / p^{2} H \cong A$ and an isomorphism $\phi: p^{\lambda} G \rightarrow p^{\lambda} H$, $\phi$ extends to an isomorphism of $G$ onto $H$. In Theorem 6.4, we find necessary and sufficient conditions for a countably generated module of finite torsion-free rank to have the $\lambda$-Zippin property. Theorem 5.3 contains a partial generalization of this to arbitrary countable Abelian groups of finite torsion-free rank.

One can define uniquely $\lambda$-elongating pairs $(A, B)$ of $Z_{p}$-modules in the obvious way, for any limit ordinal $\lambda$. In Theorem 6.5, we determine exactly what pairs $(A, B)$ of countable $Z_{p}$-modules, with $A$ of finite torsion-free rank, are uniquely $\lambda$-elongating. Surprisingly, there are such pairs in which $A$ does not have the $\lambda$-Zippin property.

We review briefly the contents of the following sections. Section 2 is a preliminary section, relating the divisibility properties of a subgroup $B$ of a group $G$ to the position of the corresponding element in the groups $\operatorname{Ext}(G / B, B)$. This provides descriptive results and existence theorems for elongations. Section 3 contains the nonuniqueness results for $\omega$-elongations of $p$-groups. Section 4 describes a family of torsion-free submodules of a mixed $Z_{p}$-module of finite torsion-free rank, the quasi-maximal torsion-free submodules. These submodules seem to have some independent interest, and may be applicable in other questions concerning mixed modules of finite torsion-free rank. Section 5 contains the positive results concerning the $\lambda$-Zippin property, and $\S 6$ contains counterexamples. Section 7 lists a number of unsolved problems.

We close this introduction by establishing some terminology. $Z_{p}$ is the ring of integers localized at $p$, and $Z_{p}^{*}$ is the ring of $p$ adic integers, the completion of $Z_{p}$. Given any Abelian group $A$, we let $A_{p}=A \otimes Z_{p}$, the localization of $A$ at $p$, which we regard as a $Z_{p}$-module. If $M$ is a $Z_{p}$-module, we let $M^{*}=M \otimes Z_{p}^{*}$, which we regard as a $Z_{p}^{*}$-module. If $G$ is any Abelian group, we define the subgroups $p^{\alpha} G$, for all ordinals $\alpha$, by $p^{\alpha+1} G=p\left(p^{\alpha} G\right)$, and, if $\alpha$ is a limit ordinal, $p^{\alpha} G=\bigcap_{\beta<\alpha} p^{\beta} G$. If $G$ is a $p$-group, then we say $G$ has length $\lambda$ if $p^{\lambda} G=0$, and $\lambda$ is the smallest ordinal with this property. 
Note that a $p$-group must be reduced to have length. By a height, we mean a formal product $\Pi_{p} p^{\lambda(p)}$ where for each prime $p, \lambda(p)$ is an ordinal or the symbol $\infty$. If $G$ is an Abelian group and $h$ a height, we define $h G=\bigcap_{p} p^{\lambda(p)} G$. We should notice that the subgroup of elements of infinite height in $G$ is exactly $h G$ where $h=$ $\Pi_{p} p^{\omega}$. Note that, for example, $2^{\alpha} G=h G$ where $h=\Pi_{p} p^{\lambda(p)}, \lambda(2)=$ $\alpha$, and $\lambda(p)=0$ for all other primes $p$. Hence it causes no difficulty to require that $\lambda(p)$ be defined for all primes $p$.

The torsion-free rank of an Abelian group $G$ is the rank of the vector space $G \otimes Q$, where $Q$ is the field of rational numbers. $G$ has finite torsion-free rank if and only if it has a finitely generated subgroup $F$ such that $G / F$ is torsion.

2. Divisibility and Ext. This is a preliminary section in which it is shown how the $p$-divisibility properties of a subgroup $B$ of a group $G$ can be determined by the corresponding element of $\operatorname{Ext}(G / B, B)$ and, in particular, in terms of the image of this element in $\operatorname{Ext}(G / B, B) / p \operatorname{Ext}(G / B, B)$. We use this analysis to deduce the existence theorems for elongations which we will need.

Lemma 2.1. If $p$ is a prime and $A$ and $B$ are arbitrary Abelian groups, the natural homomorphisms

$$
\begin{aligned}
\operatorname{Ext}(A, B / p B) & \longrightarrow \operatorname{Ext}(A[p], B / p B) \\
\operatorname{Ext}(A[p], B) & \longrightarrow \operatorname{Ext}(A[p], B / p B)
\end{aligned}
$$

are isomorphisms. All three of these groups can be naturally identified with

$$
\operatorname{Ext}(A, B) / p \operatorname{Ext}(A, B) \text {. }
$$

Proof. We first consider the last part of this lemma. R. Baer has shown [4, p. 244] that the endomorphism of $\operatorname{Ext}(A, B)$ induced by multiplication by an integer $n$ in $A$ or $B$ is again multiplication by $n$ in $\operatorname{Ext}(A, B)$. The endomorphism of $A$ given by multiplication by $p$ factors through $p A$ so we have a composition

$$
\operatorname{Ext}(A, B) \longrightarrow \operatorname{Ext}(p A, B) \longrightarrow \operatorname{Ext}(\mathrm{A}, B)
$$

where the first map is induced by the inclusion of $p A$ into $A$ and the second by multiplication by $p$. It follows that $p \operatorname{Ext}(A, B)$ can be identified with the image of $\operatorname{Ext}(p A, B)$ in $\operatorname{Ext}(A, B)$. The kernel of the map $A \rightarrow p A$ is $A[p]$ so we have a natural isomorphism

$$
\operatorname{Ext}(A, B) / p \operatorname{Ext}(A, B) \longrightarrow \operatorname{Ext}(A[p], B) \text {. }
$$


This will establish the third part of our result if we can establish the other two. Two first however, is a special case of what we have just done, replacing $B$ by $B / p B$ and remembering that $p \operatorname{Ext}(A, B / p B)=0$. We omit the proof of the second isomorphism, which is similar and is contained in [3, Theorem 26.5].

Lemma 2.2. If $p$ is a prime and $p A=p B=0$ then there is $a$ natural isomorphism $\operatorname{Ext}(A, B) \rightarrow \operatorname{Hom}(A, B)$.

We omit a detailed proof since this is [3, Theorem 26.5], but we will give an interpretation of this result. If an element in $\operatorname{Ext}(A, B)$ is represented by a short exact sequence $0 \rightarrow B \rightarrow C \stackrel{\nu}{\rightarrow}$ $A \rightarrow 0$ then the corresponding element $\phi$ of $\operatorname{Hom}(A, B)$ is defined as follows. For $x \in A$ let $y$ be an element in $C$ such that $\nu(y)=x$, and let $\phi(x)=p y$. It is easy to see that this is well defined and one can easily give a proof of the lemma in this way.

In general, if $E \in \operatorname{Ext}(A, B)$, for arbitrary Abelian groups $A$ and $B$, we let $\phi_{p}(E)$ be the element of $\operatorname{Hom}(A[p], B / p B)$ defined as follows. Suppose $E$ is represented by the short exact sequence $0 \rightarrow$ $B \rightarrow C \stackrel{\nu}{\rightarrow} A \rightarrow 0$. If $x \in A[p]$, let $y$ be any element in $G$ such that $\nu(y)=x$ and let $\phi_{p}(x)=p y+p B$.

Lemma 2.3. If $A$ and $B$ are Abelian groups, there is a natural epimorphism

$$
\operatorname{Ext}(A, B) \longrightarrow \prod_{p} \operatorname{Hom}(A[p], B / p B)
$$

associating to each $E \in \operatorname{Ext}(A, B)$ the element $\Pi_{p} \phi_{p}(E)$.

Proof. Let $S$ be the subgroup of $A$ generated by elements of prime order. There is a natural epimorphism

$$
\operatorname{Ext}(A, B) \longrightarrow \operatorname{Ext}(S, B),
$$

and since $S=\bigoplus_{p} A[p]$, a natural identification

$$
\operatorname{Ext}(S, B) \longrightarrow \prod_{p} \operatorname{Ext}(A[p], B) \text {. }
$$

By 2.1 and 2.2, there is a natural isomorphism

$$
\operatorname{Ext}(A[p], B) \longrightarrow \operatorname{Hom}(A[p], B / p B),
$$

which completes the proof.

Theorem 2.4. If $A$ and $B$ are Abelian groups and $E \in \operatorname{Ext}(A, B)$ and $E$ is represented by $0 \rightarrow B \rightarrow C \rightarrow A \rightarrow 0$, then for any $\beta>0$, 
$B \cong p^{\beta} C$ if and only if (i) $\phi_{p}(E)$ is surjective and (ii) if $K$ is the kernel of $\phi_{p}(E)$ then for every $\alpha<\beta$,

$$
A[p]=K+\left(p^{\alpha} A\right)[p] .
$$

REMARK. If $\nu: C \rightarrow A$ is the homomorphism from the above sequence, then we can identify $K=\nu(C[p])$. The condition above can be rephrased to say that $\phi_{p}(E)$ restricted to $\left(p^{\alpha} A\right)[p]$ is surjective for all $\alpha<\beta$.

Proof. Suppose first that $B \subseteq p^{\beta} C$, and $z$ is an element in $B$. Then for any $\alpha<\beta$, there is a $y \in p^{\alpha} C$ such that $p y=z$. Clearly, $\nu\left(p^{\alpha} C\right) \subseteq p^{\alpha} A$, so $\nu(y) \in\left(p^{\alpha} A \cap A[p]\right)$, and

$$
z+p B=\phi_{p}(E)(\nu(y)) .
$$

We prove the converse by induction. The surjectivity of $\phi_{p}(E)$ clearly implies that $B \subseteq p C$. We assume that for some $\gamma \leqq \beta$, we have shown that $B \cong p^{\alpha} C$ for all $\alpha<\gamma$, and we show that $B \cong p^{\gamma} C$. If $\gamma$ is a limit ordinal, this is trivial, so it suffices to show that if $B \subseteq p^{\alpha} C$ for some $\alpha<\beta$, then $B \subseteq p^{\alpha+1} C$. The induction hypothesis implies that $\nu\left(p^{\alpha} C\right)=p^{\alpha} A$. (The basic fact here is that for any $G$, if $\alpha<\gamma$, then $p^{\alpha}\left(G / p^{\gamma} G\right)=\left(p^{\alpha} G\right) /\left(p^{\gamma} G\right)$.) If $z \in B$, there is a $y \in C$ such that $\nu(y) \in\left(p^{\alpha} A\right)[p]$ and $p y-z \in p B$. Since $p^{\alpha} A=\nu\left(p^{\alpha} C\right)$, we may assume that $y \in p^{\alpha} C$. Since $p B \cong p^{\alpha+1} C$, it follows that $z \in p^{\alpha+1} C$. This completes the proof.

REMARK. If $\lambda$ is a limit ordinal, a subspace $K$ of $A[p]$ is called $\lambda$-dense if for every $\alpha<\lambda, A[p]=K+\left(p^{\alpha} A\right)[p]$. If $\lambda=\omega$, this condition is equivalent to $K$ being dense in $A[p]$ in the topology inherited from the $p$-adic topology on $A$.

Lemma 2.5. Let $V$ be a vector space, $\lambda$ a limit ordinal, and $V_{\alpha}$, $\alpha<\lambda, a$ family of subspaces indexed by ordinals less than $\lambda$, such that if $\alpha<\beta$ then $V_{\alpha} \supseteqq V_{\beta}$, and $\bigcap_{\alpha<\lambda} V_{\alpha}=0$. Then $V$ has a subspace $S$ such that for all $\alpha<\lambda, S+V_{\alpha}=V$ and such that $[V: S]=$ $\min \left(\operatorname{dim}\left(V_{\alpha}\right): \alpha<\lambda\right)$.

This was proved by Pierce [11]. If $\lambda$ is a countable ordinal, (the only case needed in this paper), it is essentially contained in [4, Lemma 31.1 and Theorem 31.4]. Other proofs of the general result are in [2] and [10].

THEOREM 2.6. Let $h=\Pi_{p} p^{\lambda(p)}$ be a height in which all of the $\lambda(p)$ are limit ordinals or 0 , and let $A$ and $B$ be Abelian groups 
such that $h A=0$. Then there is a group $G$ such that $h G \cong B$ and $G / h G \cong A$ if and only if for every prime $p$ and every ordinal $\alpha<$ $\lambda(p)$,

$$
\operatorname{dim}\left(\left(p^{\alpha} A\right)[p]\right) \geqq \operatorname{dim}(B / p B)
$$

REMARK. For $p$-groups, this was proved by Pierce in [11], and the corresponding result for modules over a discrete valuation ring is in [10, Theorem 1.6].

Proof. Assume first that the indicated inequalities hold. By 2.5, we can find subspaces $K_{p}$ of $A[p]$ (for each prime $p$ ) such that $\left[A[p]: K_{p}\right]=\operatorname{dim}(B / p B)$ and $K_{p}$ is $\lambda(p)$-dense in $A[p]$, (i.e., for every $\left.\alpha<\lambda(p), A[p]=K_{p}+\left(p^{\alpha} A\right)[p]\right)$. We can therefore find homomorphisms $\phi_{p}: A[p] \rightarrow B / p B$ which are surjective and such that $\operatorname{ker}\left(\phi_{p}\right)=$ $K_{p}$. By 2.3, there is an $E \in \operatorname{Ext}(A, B)$ such that $\phi_{p}=\phi_{p}(E)$ for all primes $p$. If this element $E$ is represented by the exact sequence $0 \rightarrow B \rightarrow G \rightarrow A \rightarrow 0$, then 2.4 implies that $G$ is a group of the desired type.

Conversely, if such an extension $E$ exists, then the homomorphisms $\phi_{p}(E)$ are surjective when restricted to $\left(p^{\alpha} A\right)[p]$, for all $\alpha<$ $\lambda(p)$ (by 2.4). Therefore,

$$
\operatorname{dim}\left(\left(p^{\alpha} A\right)[p]\right) \geqq \operatorname{dim}(B / p B)
$$

as stated.

\section{Elongations of $p$-groups.}

THEOREM 3.1. Let $A$ be a p-group with no elements of infinite height which is not a direct sum of cyclic groups. Then there exists a group $B$ with $p B=0$ and two groups $G$ and $H$ such that $p^{\omega} G \cong$ $p^{\omega} H \cong B$, and $G / p^{\omega} G \cong H / p^{\omega} H \cong A$, but such that $G$ and $H$ are not isomorphic.

Proof. Let fin. rk. $A=\min \left\{\operatorname{dim}\left(\left(p^{n} G\right)[p]\right)\right\}$. We first claim that $A$ has a pure, dense subgroup $L$ such that $\operatorname{dim}(A / L)[p]=$ fin. rk. $A$, and such that $L$ is not a direct sum of cyclic groups. This is so because $A$ has at least one pure dense subgroup $H$ such that $\operatorname{dim}(A / H)[p]=$ fin. rk. $A$ by [4, Lemma 31.1 and Theorem 31.4], from which it follows easily that $A$ is the ascending union of a sequence $H_{i}$ of such subgroups, (as in the proof of [10, Theorem 3.4]). If all of the $H_{i}$ were direct sums of cyclic groups, then $G$ would be also, by [6, Theorem 4].

Now let $L$ be the subgroup of $A$ whose existence we have just 
shown, and let $K$ be a lower basic subgroup of $A$ (i.e., a direct sum of cyclic groups such that $K$ is pure and dense and $\operatorname{dim}(A / K)[p]=$ fin. rk. $A$, [4, Theorem 31.4]). Let $B$ be a direct sum of fin. rk. $A$ cyclic groups of order $p$. By 2.3 and 2.4 there are two extensions

$$
\begin{aligned}
& E_{1}: 0 \longrightarrow B \longrightarrow G \longrightarrow A \longrightarrow 0 \\
& E_{2}: 0 \longrightarrow B \longrightarrow H \longrightarrow A \longrightarrow 0
\end{aligned}
$$

which are $\omega$-elongations of $A$ by $B$, such that $\operatorname{ker}\left(\phi\left(E_{1}\right)\right)=K[p]$, $\operatorname{ker}\left(\phi\left(E_{2}\right)\right)=L[p]$. If $G$ and $H$ were isomorphic, the isomorphism would take $K[p]$ onto $L[p]$. By the Kulikov criterion for direct sums of cyclic groups, this would imply that $L$ was a direct sum of cyclic groups which it is not, and this contradiction proves the theorem. [In detail, the Kulikov criterion says that you can tell whether a $p$-group $L$ is a direct sum of cyclic groups by looking at $L[p]$ as a metric vector space with the metric inherited from the $p$-adic metric on $L$. Since $L$ and $K$ are pure subgroups of $A$, the metrics on $L[p]$ and $K[p]$ inherited from the $p$-adic metrics of $L$ and $K$ are the same as the metrics inherited from $A$. If there were an automorphism of $A$ carrying $L[p]$ onto $K[p], L[p]$ and $K[p]$ would be isomorphic as metric vector spaces, so since $K$ is a direct sum of cyclic groups, $L$ would be also.]

Theorem 3.2. Let $A$ and $B$ be nonzero, reduced p-groups such that $A$ has no elements of infinite height and $\omega$ elongations of $A$ by $B$ exist. Suppose in addition that $A$ has cardinality c (the cardinality of the set of real numbers), and $A / p A$ is countable. Then there are $2^{c}$ nonisomorphic groups $G$ which are w-elongations of $A$ by $B$.

Remark. Since $A / p A$ is countable, $A / p^{n} A$ is countable for any positive integer $n$, which implies that $p^{n} A$, and (therefore) $\left(p^{n} A\right)[p]$ have cardinality c. It follows, in particular, that $\omega$-elongations of $A$ by $B$ exist if and only if $B / p B$ has cardinality at most c.

Proof. Let $\mathscr{C}$ be the set of all subgroups $K$ of $A[p]$ such that $K$ is dense in $A[p]$ and $[A[p]: K]=\operatorname{dim}(B / p B)$. These exist by 2.5 and the remark above. In particular, such a subgroup $C$ exists such that $[A[p]: C]=c$. The number of subgroups $K$ containing $C$ and such that $[A[p]: K]=1$ is exactly equal to the dimension of the dual space of the $Z / p Z$-vector space $A[p] / C$, so there are $2^{c}$ distinct subgroups $K$ of $A[p]$ containing $C$, such that $[A[p]: K]=1$. An easy iteration argument shows that for any cardinal $\mathfrak{n}, \mathfrak{n} \leqq \mathfrak{c}$, there are $2^{\circ}$ subgroups $K$ of $A[p]$ containing $C$ with $[A[p]: K]=\mathfrak{n}$. Hence the 
class $\mathscr{C}$ of subgroups defined above has cardinality $2^{\circ}$. For any $K \in \mathscr{C}$, we can find an element $E \in \operatorname{Ext}(A, B)$ such that $K=$ $\operatorname{ker}\left(\phi_{p}(E)\right)$ (by 2.3) and by 2.4, if this extension is represented by $0 \rightarrow B \rightarrow G \rightarrow A \rightarrow 0$, then $B=p^{\omega} G$. If for two subgroups $K$ and $L$ in $\mathscr{C}$, the corresponding extensions $G$ and $H$ are isomorphic, then an isomorphism between them induces and automorphism of $A$ carrying $K$ onto $L$.

We next observe that the automorphism group of $A$ has cardinality at most c. To see this, observe that since $A / p A$ is countable, we can find a countable subgroup $S$ of $A$ such that $A=S+p A$. Since $A / S$ is therefore divisible, $\operatorname{Hom}(A / S, A)=0$, so any endomorphism of $A$ is determined by its behavior on $S$. Since $\operatorname{Hom}(S, A)$ has cardinality at most c, the automorphism group of $A$ has cardinality at most c. We now look at our class $\mathscr{C}$ of subgroups of $A[p]$ defined above and think of two of them as equivalent if there is an automorphism of $A$ carrying one onto the other. Since there are $2^{\mathrm{c}}$ elements of $\mathscr{C}$ and at most $\mathrm{c}$ automorphisms, there are $2^{\mathrm{c}}$ equivalence classes, which completes the proof of the theorem.

Corollary 3.3. Let $A$ and $B$ be p-groups such that $A$ has no elements of infinite height, $A / p A$ is countable, $B \neq p B$, and such that $\omega$-elongations of $A$ by $B$ exist. Then if $A$ is not a direct sum of cyclic groups, the continuum hypothesis implies that there are $2^{c}$ nonisomorphic $\omega$-elongations of $A$ by $B$.

Proof. If $A / p A$ is countable, $A$ is a direct sum of cyclic groups if and only if $A$ is countable. If $A$ is not a direct sum of cyclic groups, therefore, $A$ is not countable, and the continuum hypothesis implies that $A$ has cardinality $c$, and the result follows from 3.2.

The first example of two nonisomorphic $p$-groups $G$ and $H$ such that $p^{\omega} G \cong p^{\omega} H$ and $G / p^{\omega} G \cong H / p^{\omega} H$, was given by Kulikov [4, §39, C]. This rather special example is the only one previously published. In unpublished work, Crawley showed that if $G$ is a reduced $p$-group such that $p^{\omega} G \neq 0$ and $G / p^{\omega} G$ is torsion-complete, then there is group $H$, not isomorphic to $G$, such that $p^{\omega} G \cong p^{\omega} H$ and $G / p^{\omega} G \cong H / p^{\omega} H$.

Theorem 3.2 was proved in 1966 [16], and takes care of $p$-groups with countable basic subgroups (assuming the continuum hypothesis). Theorem 3.1 is the most satisfactory available for groups with uncountable basic subgroups, (though it does not include Crawley's examples), but it is still less satisfactory than 3.2 in that it does not solve the question of uniquely $\omega$-elongating pairs. The author is indebted to R. Nunke for showing him how a rather cumbersome result which the author proved in 1970 could be combined with Hill's theorem to yield 3.1 . 


\section{Quasi-maximal torsion-free submodules.}

THEOREM 4.1. Let $M$ be a $Z_{p}$-module of finite torsion-free rank and $\alpha$ an ordinal such that $t(M)$ has length $\alpha$. Then $M$ has $a$ torsion-free submodule $F$ such that $M / F$ is torsion and $p^{\alpha}(M / F)=0$.

REMARK. It is fairly easy to obtain a torsion-free subgroup such that $M / F$ is torsion and reduced, but, in general, the length of $M / F$ could still be any ordinal less than $\alpha+\omega$, even if $p^{\alpha} M=0$. This arises from the existence of "slack" modules, a phenomenon discussed in detail, with examples, in [12].

Proof. For any $Z_{p}$-module $A$, we let $A^{*}=A \otimes Z_{p}^{*}$. Let $L$ be a free submodule of $M$ such that $M / L$ is torsion. By replacing $L$ by $p^{n} L$ for some $n$, if necessary, we may assume that $L^{*} \cap p^{\alpha} M^{*}$ is a summand of $L$. We now let $F=\left\{x \in M: x+L \in p^{\alpha}(M / L)\right\}$. We must show that $F$ is torsion-free.

We remind the reader that a submodule of $X$ of a module $N$ is nice if for all ordinals $\beta, p^{\beta}(N / X)=\left(p^{\beta} N+X\right) / X$. It is well known that if $N$ is a module over a complete discrete valuation ring, then any finitely generated submodule is nice. It follows that $L^{*}$ is nice in $M^{*}$. Therefore, if $x \in F^{*}, x+L^{*} \in p^{\alpha}\left(M^{*} / L^{*}\right)$, so $x+L^{*}=y+L^{*}$ for some $y \in p^{\alpha} M^{*}$. Hence, $x=y+z$ with $y \in p^{\alpha} M^{*}, z \in L^{*}$. If $x$ were torsion, we would have $p^{n} z \in p^{\alpha} M^{*}$ for some $n$, from which, by our previous hypothesis, $z \in p^{\alpha} M^{*}$, so $x \in p^{\alpha} M^{*}$. Since $p^{\alpha} M^{*}$ is torsion-free, $x=0$.

THEOREM 4.2. If $M$ is a reduced $Z_{p}$-module of finite torsion-free rank and $F$ a torsion-free submodule the following properties of $F$ are equivalent:

(i) $M / F$ is reduced and torsion,

(ii) for all torsion-free modules $G$ of finite rank and homomorphisms $f: G \rightarrow M$, there is an integer $n$ such that $p^{n} f(G) \leqq F$.

Proof. If $G$ is a torsion-free module of finite rank then any reduced torsion factor is finite. This immediately implies that if $F$ satisfies condition (i) then it satisfies condition (ii). Conversely, suppose (ii) holds for $F$ and that $F_{0}$ is the torsion-free submodule whose existence is given by 4.1. Since $M / F_{0}$ is reduced and torsion, so is $M / p^{n} F_{0}$, for any $n$. If $p^{n} F_{0} \subseteq F$, then $M / F$ is a quotient of a reduced torsion group with finite kernel, so $M / F$ is also reduced and torsion.

Definition. If $M$ is a reduced $Z_{p}$-module of finite torsion-free 
rank, a submodule $F$ satisfying the conditions of Theorem 4.2 is called a quasi-maximal torsion-free submodule.

Theorem 4.1 implies that quasi-maximal torsion-free submodules exist. It is easy to give examples to show that a maximal torsionfree submodule need not be quasi-maximal in this sense. If $A$ and $B$ are torsion-free submodules of a module $M$, we say that $A$ is quasi-contained in $B$ if for some $N, p^{n} A \subseteq B$. Clearly, a torsion-free submodule of $M$ is quasi-maximal (in the sense of the above definition) if and only if every torsion-free submodule is quasi contained in it. Two quasi-maximal torsion-free submodules of $M$ need not be isomorphic, but they are clearly quasi-isomorphic, and the operation associating to each reduced module $M$ of finite torsion-free rank the quasi-equivalence class of its quasi-maximal torsion-free submodules is functorial. For details the category in which this functor takes its values, we refer to [14]. The functorial properties of this operation are not needed in this paper.

CoRollary 4.3. The following conditions on a reduced $Z_{p}$-module of finite torsion-free rank are equivalent:

(i) every torsion-free submodule of $M$ is free;

(ii) $M$ has a free submodule $F$ such that $M / F$ is reduced and torsion;

(iii) $M \otimes Z_{p}^{*}$ is a reduced $Z_{p}^{*}$-module.

Proof. (i) implies (ii) by 4.2. If (ii) holds, we look at the exact sequence $0 \rightarrow F \rightarrow M \rightarrow M / F \rightarrow 0$ and tensor with $Z_{p}^{*}$. $(M / F) \otimes Z_{p}^{*} \cong$ $M\left\langle F\right.$, and $F \otimes Z_{p}^{*}$ is a free $Z_{p}^{*}$-module, so $M \otimes Z_{p}^{*}$ is the extension of two reduced $Z_{p}^{*}$-modules, and is therefore reduced. Finally, if (iii) holds and $A$ is a torsion-free submodule of $M$, then $A \otimes Z_{p}^{*}$ is necessarily reduced, and therefore (by [9, Theorem 20]) $A \otimes Z_{p}^{*}$ is free. If the rank of $A$ is $k$, this implies that the rank of $A / p A$ is also $k$ (since $A \otimes Z_{p}^{*}$ is free on $k$ generators and $A / p A \cong$ $\left(A \otimes Z_{p}^{*}\right) / p\left(A \otimes Z_{p}^{*}\right)$.) By [17, Corollary 8], this implies that $A$ is free.

5. Unique elongations of modules and groups of finite rank.

Lemma 5.1. Let $A$ and $B$ be $Z_{p}$-modules, $S$ and $T$ submodules such that $A / S$ and $B / T$ are torsion, and $f: S \rightarrow T$ an isomorphism. If the induced isomorphism $f^{*}: S^{*} \rightarrow T^{*}$ extends to an isomorphism $g: A^{*} \rightarrow B^{*}$, then $f$ extends to an isomorphism of $A$ onto $B$. Similarly, if $A$ and $B$ are Abelian groups, $S$ and $T$ subgroups such that $A / S$ and $B / T$ are torsion, and $f: S \rightarrow T$ an isomorphism and if for each prime $p$ the isomorphism $f_{p}: S_{p} \rightarrow T_{p}$ extends to an isomorphism of $A_{p}$ onto $B_{p}$, then $f$ extends to an isomorphism of $A$ onto $B$. 
Proof. For the first statement, we note that $A$ and $B$ are imbedded in $A^{*}$ and $B^{*}$ respectively with torsion-free divisible cokernels. It follows that we can identify $A$ with the set of those elements $x \in A^{*}$ such that for some $n, p^{n} x \in S$, and we can identify $B$ similarly in $B^{*}$. Hence, the restriction of $g$ to $A$ takes $A$ isomorphically onto $B$.

For the second statement, let $P(A)=\Pi_{p} A_{p}$ and, again, note that $A$ can be imbedded in $P(A)$ with torsion-free divisible cokernel, (by a routine computation). The rest of the proof is the same as before.

THEOREM 5.2. Let $A$ be a countable reduced $Z_{p}$-module of finite torsion-free rank, and $\lambda$ a limit ordinal such that $p^{\lambda} t(A)=0 . \quad$ Let $G$ and $H$ be modules and $X$ and $Y$ submodules of $p^{\lambda} G$ and $p^{\lambda} H$, respectively such that $G / X \cong H / Y \cong A$ and let $\phi: X \rightarrow Y$ be an isomorphism. If the reduced part of $X$ has bounded order, or if all torsion-free submodules of $A$ are free, then $\phi$ extends to an isomorphism of $G$ onto $H$.

Proof. We assume that the above isomorphisms induce surjective homomorphisms $g: G \rightarrow A, g_{0}: H \rightarrow A$ with kernels $X$ and $Y$. Choose a torsion-free submodule $F$ of $A$ such that $A / F$ is torsion and $p^{\lambda}(A / F)=0$ (as we may, by 4.1). Let $G^{\prime}=g^{-1}\left(F^{\prime}\right), H^{\prime}=g_{0}^{-1}(F)$. By hypothesis, $\operatorname{Ext}(F, X)=0$, so there are submodules $M$ and $N$ of $G$ and $H$ respectively such that $G^{\prime}=M \oplus X, H^{\prime}=N \oplus Y$. We obtain an isomorphism $\phi^{\prime}: G^{\prime} \rightarrow H^{\prime}$ by defining $\phi^{\prime}=\phi$ on $X$ and defining $\phi^{\prime}$ on $M$ so that $g_{0} \phi^{\prime}=g$.

We want to use Hill's extension theorem [5], in the form stated and proved by Walker [15], to extend $\phi^{\prime}$ to an isomorphism of $G$ onto $H$. Hill's theorem cannot be applied directly because $F$ is not necessarily a nice submodule of $A$. We recall that a submodule $Z$ of $G$ is nice if for all ordinals $\alpha, p^{\alpha}(G / Z)=\left(Z+p^{\alpha} G\right) / Z$. In order to have lots of nice submodules, it is desirable to work with modules over $Z_{p}^{*}$.

We first claim that $\left(G^{\prime}\right)^{*}$ and $\left(H^{\prime}\right)^{*}$ are nice submodules of $G^{*}$ and $H^{*}$ respectively. $\left(G^{\prime}\right)^{*}=X^{*} \oplus M^{*}$ and $M^{*}=D \oplus E$, where $D$ is divisible and $E$ is finitely generated. It is clear (by 2.4 for example) that $X^{*} \subseteq p^{\lambda} G^{*}$. Since $p^{\lambda}\left(G^{*} /\left(G^{\prime}\right)^{*}\right)=0$ (by our hypothesis on $A / F), p^{\lambda} G^{*} \subseteq\left(G^{\prime}\right)^{*}$. Therefore, $\left(G^{\prime}\right)^{*}=p^{\lambda} G^{*}+E$. For modules over a complete discrete valuation ring, the the sum of a nice submodule and a finitely generated submodule is again nice, so $\left(G^{\prime}\right)^{*}$ and $\left(H^{\prime}\right)^{*}$ are nice submodules of $G^{*}$ and $H^{*}$.

We next must show that the induced isomorphism $\left(\phi^{\prime}\right)^{*}:\left(G^{\prime}\right)^{*} \rightarrow$ $\left(H^{\prime}\right)^{*}$ is height preserving. By construction, $\left(\phi^{\prime}\right)^{*}$ preserves the 
heights of elements of height less than $\lambda$, and so does the inverse of $\left(\phi^{\prime}\right)^{*}$. It follows that $\left(\phi^{\prime}\right)^{*}$ takes $p^{\lambda} G^{*}$ isomorphically onto $p^{\lambda} H^{*}$. If $x \in p^{\lambda} G^{*}$, then the height of $x$ in $G^{*}$ can be computed from its height in $p^{\lambda} G^{*}$. Using this and the corresponding fact for $p^{2} H^{*}$, we can see that the restriction of $\left(\phi^{\prime}\right)^{*}$ to $p^{\lambda} G^{*}$ is height preserving. Since we already know $\left(\phi^{\prime}\right)^{*}$ preserves heights less than $\lambda,\left(\phi^{\prime}\right)^{*}$ is height preserving.

We now have two modules $G^{*}$ and $H^{*}$, with the same Ulm invariants, nice submodules $\left(G^{\prime}\right)^{*}$ and $\left(H^{\prime}\right)^{*}$ such that $G^{*} /\left(G^{\prime}\right)^{*}$ and $H^{*} /\left(H^{\prime}\right)^{*}$ are countable torsion modules, and a height preserving isomorphism $\left(\phi^{\prime}\right)^{*}:\left(G^{\prime}\right)^{*} \rightarrow\left(H^{\prime}\right)^{*}$. Hill's extension theorem [5], in the form stated by Walker [15], enables us to extend $\left(\phi^{\prime}\right)^{*}$ to an isomorphism of $G^{*}$ onto $H^{*}$. (The statement of Hill's theorem involves a condition on relative Ulm invariants. We should remark that since $\left(G^{\prime}\right)^{*}=E+p^{\lambda} G^{*}$ and $\left(H^{\prime}\right)^{*}=E_{0}+p^{\lambda} H^{*}$, where $E$ and $E_{0}$ are finitely generated, it is trivial to verify that the relative Ulm invariants of $\left(G^{\prime}\right)^{*}$ and $\left(H^{\prime}\right)^{*}$ in $G$ and $H$ are the same.) Lemma 5.1 allows us to conclude that the original isomorphism $\phi: X \rightarrow Y$ extends to an isomorphism of $G$ onto $H$, as desired.

Definition. If $h=\Pi_{p} p^{\lambda(p)}$ is a height in which $\lambda(p)$ is a limit ordinal or 0 for each prime $p$, and $A$ is an Abelian group with $h A=$ 0 , we say $A$ has the $h$-Zippin property if whenever we are given groups $G$ and $H$ such that $G / h G \cong H / h H \cong A$, and an isomorphism $\phi: h G \rightarrow h H, \phi$ extends to an isomorphism of $G$ onto $H$.

THEOREM 5.3. Let $A$ be a countable Abelian group of finite torsion-free rank and $h=\Pi_{p} p^{\lambda(p)}$ a height in which each $\lambda(p)$ is a limit ordinal or 0 , and such that $h A=0$. Let $\Gamma$ be the set of primes for which $t(A)_{p}$ has length $\lambda(p)$. Suppose $A$ has a free subgroup $F$ such that $A / F$ is torsion and $p^{\lambda(p)}(A / F)_{p}=0$ for all $p \in \Gamma$. Then $A$ has the h-Zippin property.

Proof. Let $G$ and $H$ be groups, $\phi: h G \rightarrow h H$ an isomorphism, and suppose that $G / h G \cong H / h H \cong A$. Let $g: G \rightarrow A$, and $g_{0}: H \rightarrow A$ be the induced homomorphisms, and let $G^{\prime}=g^{-1}(F), H^{\prime}=g_{0}^{-1}(F)$. Choose splittings $G^{\prime}=h G \oplus M, H^{\prime}=h H \oplus N$, and let $\phi^{\prime}: G^{\prime} \rightarrow H^{\prime}$ be the isomorphism induced in the obvious way. The argument of 5.2 applies to show that $\phi_{p}^{\prime}: G_{p}^{\prime} \rightarrow H_{p}^{\prime}$ extends to an isomorphism of $G_{p}$ onto $H_{p}$, if $p \in \Gamma$. If $p \notin \Gamma, h G$ and $h H$ are necessarily $p$-divisible, so $(h G)_{p}$ and $(h H)_{p}$ are divisible, and, therefore, summands of $G_{p}$ and $H_{p}$ respectively. By a standard argument, the complements of $(h G)_{p}$ and $(h H)_{p}$ in $G_{p}$ and $H_{p}$ may be assumed to contain $M_{p}$ and $N_{p}$ respectively. These complements are both naturally isomorphic to 
$A_{p}$, so, clearly, $\phi_{p}^{\prime}$ extends to an isomorphism of $G_{p}$ onto $H_{p}$ if $p \notin \Gamma$. An application of 5.1 completes the proof of the theorem.

EXAMPLE. Suppose $h=\Pi_{p} p^{\omega}$, so that $h G$ is the subgroup of elements of infinite height in $G$, and that $A=S \oplus T$, where $S$ is torsion-free of finite rank and $T$ is torsion. Then 5.3 says that $A$ has the $h$-Zippin property if $S_{p}$ is a free $Z_{p}$-module for all $p$ such that $T_{p}$ is unbounded. In particular, if $S$ is the subgroup of rational numbers generated by the elements $p^{-1}$ for all primes $p$, then $S \oplus$ $T$ has the $h$-Zippin property (for this $h$ ) for any unbounded torsion group $T$. (In the case in which $S$ is free, this result is in [8, Theorem 3.7].) It will be a consequence of the results of the next section that if $S_{p}$ is not free for some $p$ such that $T_{p}$ is unbounded, then $A$ does not have the $h$-Zippin property.

\section{Nonuniqueness theorems.}

Lemma 6.1. If $N$ is a countable, reduced $Z_{p}$-module which is not of bounded order, and $M$ is a torsion-free $Z_{p}$-module of finite rank, then either $M$ is free or $\operatorname{Ext}(M, N)$ has an uncountable, divisible, torsion-free summand.

Proof. By [17, Lemma 3] we can find a free submodule $F$ of $M$ such that $M / F$ is nonzero, torsion-free and divisible (assuming that $M$ is not free). We consider the exact sequence

$(*) \operatorname{Hom}(F, N) \longrightarrow \operatorname{Ext}(M / F, N) \longrightarrow \operatorname{Ext}(M, N) \longrightarrow 0$.

$\operatorname{Ext}(M / F, N)$ is a direct sum of a finite number of copies of $\operatorname{Ext}(Q, N)$. It is routine that $\operatorname{Ext}(Q, N)$ is torsion-free and divisible. The exact sequence $0 \rightarrow \operatorname{Hom}\left(Z_{p}, N\right) \rightarrow \operatorname{Ext}\left(Q / Z_{p}, N\right) \rightarrow \operatorname{Ext}(Q, N) \rightarrow$ 0 exhibits $\operatorname{Ext}(Q, N)$ as a quotient of the group $\operatorname{Ext}\left(Q / Z_{p}, N\right)$ with countable kernel. We claim that $\operatorname{Ext}\left(Q / Z_{p}, N\right)$ is uncountable, and, therefore, that $\operatorname{Ext}(Q, N)$ is uncountable. $\operatorname{Ext}\left(Q / Z_{p}, N\right)$ is the cotorsion hull of $N$, and hence is torsion if and only if $N$ is of bounded order. Since this is not the case, $\operatorname{Ext}\left(Q / Z_{p}, N\right)$ is not torsion, and since it is a module over the ring of $p$-adic integers, it is necessarily uncountable. Returning to the sequence $(*)$, this shows that $\operatorname{Ext}(M / F, N)$ is an uncountable, torsion-free, divisible group. Since Hom $(F, N)$ is countable, its image is contained in a countable summand of $\operatorname{Ext}(M / F, N)$, so $\operatorname{Ext}(M, N)$ is the direct sum of a countable group and an uncountable torsion-free divisible group, as claimed.

We include the next lemma to clarify what it means for an element of $\operatorname{Ext}(A, B)$ to have infinite order. 
LEMMA 6.2. If $E$ is an element of $\operatorname{Ext}(A, B)$ represented by an exact sequence $0 \rightarrow B \rightarrow G \rightarrow A \rightarrow 0$, then $n E=0$ if and only if the sequence restricted to $n A$ splits (i.e., if $\phi: G \rightarrow A$ is the homomorphism of the sequence, then there is an $f: n A \rightarrow G$ such that $\phi f$ is the identity on $n A$ ).

This is a consequence of Baer's theorem [4, p. 244] that multiplication by $n$ in $A$ induces multiplication by $n$ in $\operatorname{Ext}(A, B)$. For a detailed discussion and interpretation, we refer to [13].

THEOREM 6.3. If $A$ and $B$ are reduced, countable $Z_{p}$-modules, and $\lambda$ a limit ordinal such that (i) $p^{\lambda} A=0$, (ii) $t(A)$ has length $\lambda$, (iii) $A$ has finite torsion-free rank, (iv) $A$ has a torsion-free submodule which is not free, and (v) $B$ is not of bounded order, then there are nonisomorphic modules $M$ and $N$ such that $p^{\lambda} M \cong p^{\lambda} N \cong$ $B$ and $M / p^{2} M \cong N / p^{2} N \cong A$.

Proof. Let $S$ be a quasi-maximal torsion-free submodule of $A$, and $T$ the torsion submodule of $A$. Consider the surjective homomorphism

$$
\operatorname{Ext}(A, B) \longrightarrow \operatorname{Ext}(T \oplus S, B)=\operatorname{Ext}(T, B) \oplus \operatorname{Ext}(S, B) .
$$

Whether or not an element $E$ of $\operatorname{Ext}(A, B)$ corresponds to a $\lambda$ elongation of $A$ by $B$ depends only on the image of $E$ in $\operatorname{Ext}(T, B)$, by 2.4. Choose $E \in \operatorname{Ext}(A, B)$ such that $E$ corresponds to a $\lambda$-elongation of $A$ by $B$, (as we may, by 2.6), and such that the image of $E$ in $\operatorname{Ext}(S, B)$ is 0 . Suppose that $E^{\prime}$ is another element of $\operatorname{Ext}(A, B)$ corresponding to a $\lambda$-elongation of $A$ by $B$, and that $E$ and $E^{\prime}$ are represented by exact sequences

$$
\begin{aligned}
& 0 \longrightarrow B \longrightarrow M \longrightarrow A \longrightarrow 0 \\
& 0 \longrightarrow B \longrightarrow N \longrightarrow A \longrightarrow 0 .
\end{aligned}
$$

If $M$ and $N$ are isomorphic, an isomorphism from $N$ to $M$ induces an automorphism $g$ of $A$. By 4.2, there is an integer $n$ such that $g\left(p^{n} S\right) \subseteq S$. Since the restriction of $E$ to $S$ splits, it follows that the restriction of $E^{\prime}$ to $p^{n} S$ splits. By 6.2 it follows that the image of $E^{\prime}$ in $\operatorname{Ext}(S, B)$ is a torsion element. On the other hand, by 6.1 we can clearly choose an $E^{\prime} \in \operatorname{Ext}(A, B)$ which represents a $\lambda$ elongation of $A$ by $B$ and such that the image of $E^{\prime}$ in $\operatorname{Ext}(S, B)$ is not a torsion element, from which it follows that there is a $\lambda$ elongation of $A$ by $B$ which is not isomorphic to $M$.

Corollary 6.4. If $A$ is a countable $Z_{p}$-module of finite torsionfree rank and $\lambda$ a limit ordinal such that $p^{\lambda} A=0$ and $t(A)$ has 
length $\lambda$, then $A$ has the $\lambda$-Zippin property if and only if all torsionfree submodules of $A$ are free.

This is a corollary of 5.2 and 6.3. 4.3 contains alternative forms of the condition on $A$.

CoRollary 6.5. If $(A, B)$ is a pair of countable, reduced $Z_{p^{-}}$ modules and $\lambda$ is a limit ordinal, such that $B \neq 0, p^{\lambda} A=0, t(A)$ has length $\lambda$, and $A$ has finite torsion-free rank, then $(A, B)$ is uniquely $\lambda$-elongating pair if and only if either (i) $B$ has bounded order, or (ii) $A$ has the $\lambda$-Zippin property.

We remark that this result does not carry over directly to modules over an arbitrary discrete valuation ring. We also remark that other uniquely $\lambda$-elongating pairs can be obtained by allowing $B$ to be uncountable - for example, $B$ could be any cotorsion module such that $B / p B$ is countable.

\section{Some unsolved problems.}

Problem 1. Find all pairs $(A, B)$ of $p$-groups which are uniquely $\omega$-elongating. In particular, if $A$ is an unbounded $p$-group with no elements of infinite height such that any two $\omega$-elongations of $A$ by $Z / p Z$ are isomorphic, is $A$ necessarily a direct sum of cyclic groups? (This question was raised long ago by Peter Crawley.)

Problem 2. Find an analogue of the theory of quasi-maximal torsion-free submodules for Abelian groups of finite torsion-free rank. In general, there is not a maximal quasi-isomorphism class of torsion-free subgroups, but one might obtain one by restricting attention to "quotient-divisible" groups.

Problem 3. Find the appropriate generalizations of the results of $\S 5$ and 6 for countable $Z_{p}$-modules of infinite rank.

PROBLEM 4. Find interesting results which hold without countability restrictions. Many of the positive results generalize trivially to modules whose torsion submodules are totally projective. Do they hold if one only requires the torsion submodules to be $S$-groups? This is suggested by Nunke's results [10], which also illustrate the pathology which can arise in the uncountable case.

Problem 5. Find the right generalizations of 6.4 and 6.5 to arbitrary countable Abelian groups with finite torsion-free rank. Even for the height $h=\Pi_{p} p^{\omega}$, this is an interesting open problem. 
It is easy to see that if $A$ has the $h$-Zippin property for some $h$ of the type used in 5.3, and if $p \in \Gamma$, (still using the terminology of 5.3), then every torsion-free submodule of $A_{p}$ must be free. However, there is still some distance between this necessary condition and the sufficient condition of 5.3. Under some additional hypotheses, these conditions may coincide - for example, if $A=T \oplus S$ where $T$ is torsion and $S$ is torsion-free.

\section{REFERENCES}

1. P. Crawley, Abelian p-groups determined by their Ulm sequences, Pacific, J. Math., 22 (1967), 235-239.

2. D. O. Cutler and P. F. Dubois, Generalized final rank for arbitrary limit ordinals, Pacific. J. Math., 37 (1971), 345-351.

3. S. Eilenberg and S. MacLane, On the groups $H(\pi, n)$, II, Ann. Math., 60 (1954), 49-139.

4. L. Fuchs, Abelian Groups, Pergamon, Oxford, 1960.

5. P. Hill, The classification of Abelian groups, 1968 lecture notes.

6. - On the freeness of Abelian groups, a generalization of Pontryagin's theorem, Bull. Amer. Math. Soc., 76 (1970), 1118-1120.

7. P. Hill and C. Megibben, Extending automorphisms and lifting decompositions in Abelian groups, Math. Ann., 175 (1968), 159-168.

8. - On direct sums of countable groups and generalizations, "Études sur les groupes Abéliens", ed. B. Charles, Dunod, Paris, 1968, 183-206.

9. I. Kaplansky, Infinite Abelian Groups, revised edition, Ann Arbor, U. of Mich., 1969.

10. R. Nunke, Uniquely elongating modules, to appear in the proceedings of the Nov. 1972 Rome conference on Abelian groups.

11. R. S. Pierce, Abelian Group Seminar Notes, New Mexico State University, 1968.

12. A. E. Stratton, Mixed modules over an incomplete discrete valuation ring, Proc. London Math. Soc., (3) 21 (1970), 201-218.

13. C. Walker, Properties of Ext and quasi-splitting of Abelian groups, Acta Math. Acad. Sci. Hung., 15 (1964), 724-732.

14. E. A. Walker, Quotient categories and quasi-isomorphisms of Abelian groups, Proc. Colloq. Abelian Groups (Tihany), Akad. Kiado, Budapest, 1964, pp. 147-162.

15. Ulm's theorem for totally projective groups, Proc, Amer. Math. Soc., 37 (1973), 387-392.

16. R. B. Warfield, Jr., Nonisomorphic Abelian groups with isomorphic Ulm factors, Notices Amer. Math. Soc., 14 (1967), 276.

17. - Homomorphisms and duality for torsion-free groups, Math. Zeit., 107 (1968), 189-200.

18. L. Zippin, Countable torsion groups, Ann. Math., 36 (1935), 86-99.

Received September 11, 1973. Research supported by N.S.F. Grant GP-28946.

UNIVERSITY OF WASHINGTON 


\section{PACIFIC JOURNAL OF MATHEMATICS}

EDITORS

RICHARD ARENS (Managing Editor)

University of California

Los Angeles, California 90024

R. A. Beaumont

University of Washington

Seattle, Washington 98105

J. DugundJI

Department of Mathematics

University of Southern California

Los Angeles, California 90007

D. Gilbarg and J. Milgram

Stanford University

Stanford, California 94305

\section{ASSOCIATE EDITORS}

E. F. BECKENBACH

B. H. NeumanN

F. WOLF

K. YOSHIDA

\section{SUPPORTING INSTITUTIONS}

UNIVERSITY OF BRITISH COLUMBIA CALIFORNIA INSTITUTE OF TECHNOLOGY

UNIVERSITY OF CALIFORNIA

MONTANA STATE UNIVERSITY

UNIVERSITY OF NEVADA

NEW MEXICO STATE UNIVERSITY

OREGON STATE UNIVERSITY

UNIVERSITY OF OREGON

OSAKA UNIVERSITY

\author{
UNIVERSITY OF SOUTHERN CALIFORNIA \\ STANFORD UNIVERSITY \\ UNIVERSITY OF TOKYO \\ UNIVERSITY OF UTAH \\ WASHINGTON STATE UNIVERSITY \\ UNIVERSITY OF WASHINGTON \\ $\stackrel{*}{*} \stackrel{*}{*}{ }^{*}{ }^{*}{ }^{2}$ AMERICAN MATHEMATICAL SOCIETY \\ NAVAL WEAPONS CENTER
}




\section{Pacific Journal of Mathematics}

\section{Vol. 52, No. $1 \quad$ January, 1974}

David R. Adams, On the exceptional sets for spaces of potentials ............ 1

Philip Bacon, Axioms for the Čech cohomology of paracompacta ............ 7

Selwyn Ross Caradus, Perturbation theory for generalized Fredholm operators ..... 11

Kuang-Ho Chen, Phragmén-Lindelöf type theorems for a system of nonhomogeneous equations ............................ 17

Frederick Knowles Dashiell, Jr., Isomorphism problems for the Baire classes .......

M. G. Deshpande and V. K. Deshpande, Rings whose proper homomorphic images are right subdirectly irreducible . . . . . . . . . . . . . . . . . . . . . . . . .

Mary Rodriguez Embry, Self adjoint strictly cyclic operator algebras .............

Paul Erdős, On the distribution of numbers of the form $\sigma(n) / n$ and on some related

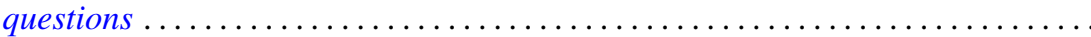

Richard Joseph Fleming and James E. Jamison, Hermitian and adjoint abelian

operators on certain Banach spaces ............................

Stanley P. Gudder and L. Haskins, The center of a poset .................. 85

Richard Howard Herman, Automorphism groups of operator algebras . . . ........

Worthen N. Hunsacker and Somashekhar Amrith Naimpally, Local compactness of families of continuous point-compact relations ....................

Donald Gordon James, On the normal subgroups of integral orthogonal groups ....

Eugene Carlyle Johnsen and Thomas Frederick Storer, Combinatorial structures in

loops. II. Commutative inverse property cyclic neofields of prime-power

order.

Ka-Sing Lau, Extreme operators on Choquet simplexes . . . . . . . . . . . . . . 129

Philip A. Leonard and Kenneth S. Williams, The septic character of 2, 3, 5 and $7 \ldots 143$

Dennis McGavran and Jingyal Pak, On the Nielsen number of a fiber map ........ 149

Stuart Edward Mills, Normed Köthe spaces as intermediate spaces of $L_{1}$ and

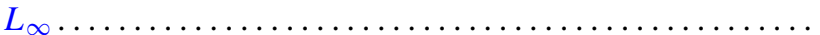

Philip Olin, Free products and elementary equivalence. .

Louis Jackson Ratliff, Jr., Locally quasi-unmixed Noetherian rings and ideals of the principal class.

Seiya Sasao, Homotopy types of spherical fibre spaces over spheres ...

Helga Schirmer, Fixed point sets of polyhedra ...

Kevin James Sharpe, Compatible topologies and continuous irreducible

representations.

Frank Siwiec, On defining a space by a weak base . . . . . . . . . . . . . . . 233

James McLean Sloss, Global reflection for a class of simple closed curves ....... 247

M. V. Subba Rao, On two congruences for primality . .

Raymond D. Terry, Oscillatory properties of a delay differential equation of even

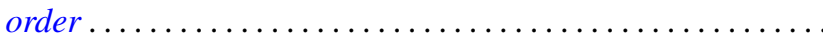

Joseph Dinneen Ward, Chebyshev centers in spaces of continuous functions . .

Robert Breckenridge Warfield, Jr., The uniqueness of elongations of Abelian

groups...

V. M. Warfield, Existence and adjoint theorems for linear stochastic differential

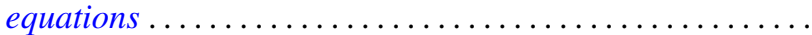

